\title{
Thickness of softened human enamel removed by toothbrush abrasion: an in vitro study
}

\author{
J. Voronets • A. Lussi
}

Received: 24 September 2008 / Accepted: 18 May 2009/Published online: 5 June 2009

(C) Springer-Verlag 2009

\begin{abstract}
The aim of the study was to assess the thickness of softened enamel removed by toothbrushing. Human enamel specimens were indented with a Knoop diamond. Softening was performed with citric acid or orange juice. The specimens were brushed in a brushing machine with a manual soft toothbrush in toothpaste slurry or in artificial saliva. Enamel loss was calculated from the change in indentation depth of the same indent before and after abrasion. Mean surface losses (95\% confidence interval) were recorded in treatment groups (in nanometers): (1) citric acid, abrasion with slurry $=339$ (280-398); (2) citric acid, abrasion with artificial saliva $=16(5-27)$; (3) orange juice, abrasion with slurry $=268(233-303)$; (4) orange juice, abrasion with artificial saliva $=14(5-23)$; (5) no softening, abrasion with slurry $=28(10-46)$. The calculated thickness of the softened enamel varied between 254 and $323 \mathrm{~nm}$, depending on the acid used.
\end{abstract}

Keywords Erosion · Toothbrush abrasion · Citric acid . Orange juice $\cdot$ Toothpaste slurry

\section{Introduction}

Erosion is the result of the contact between the dental tissues and a solution which is low in $\mathrm{pH}$, either after the consumption of extrinsic acidic fluids or when gastric fluids come into the oral cavity [1]. Chemical erosion of the teeth occurs either by the hydrogen ion derived from acids or by anions which can bind or be complexed with calcium. The

J. Voronets $(\bowtie) \cdot$ A. Lussi

Department of Preventive, Restorative and Pediatric Dentistry,

University of Bern,

Freiburgstrasse 7,

3010 Bern, Switzerland

e-mail: voronets.julia@sunrise.ch hydrogen ions are derived from acids as they dissociate in water and can attack the tooth mineral crystals and directly dissolve by combining with either the carbonate ion or the phosphate ion. Acids such as citric acid have a more complex interaction. In water, they exist as a mixture of hydrogen ions, acid anions (e.g., citrate), and undissociated acid molecules. The hydrogen ion behaves exactly as described above and directly attacks the crystal surface. Over and above the effect of the hydrogen ion, the citrate anion may be complexed with calcium-also removing it from the crystal surface. However, the full chelating capacity does not occur in acidic $\mathrm{pH}$ [2]. In a white spot caries lesion, the decalcification has occurred below the surface and the lesion is coved by a virtually intact surface zone of enamel. There is clinical evidence that such lesions can be remineralized because saliva and plaque fluid are normally supersaturated with respect to tooth mineral. Furthermore, the subsurface lesion provides a suitable matrix for crystal growth after calcium and phosphate ions have passed through the pellicle and surface enamel. In the case of erosion, enamel is exposed to a solution undersaturated with respect to both hydroxyapatite and fluorapatite. The enamel is dissolved without the formation of any mineral on the surface enamel and no surface layer remains to cover a subsurface body of lesion. The enamel is dissolved layer by layer and it cannot be measurably recalcified because there is no suitable matrix for crystal growth [3, 4].

However, in most clinical cases, it is not only erosion which is responsible for tooth wear. It acts in synergy with abrasion and attrition. If there is no erosion, it is unlikely that great surface loss occurs. It is believed that excessive toothbrushing in vivo might remove the demineralised enamel nearly completely. An in situ study showed that the mean surface loss of softened human enamel specimens after abrasion amounted to $258 \mathrm{~nm}$ [5]. In an in vitro study under controlled conditions, the depth of briefly eroded 
enamel amounted to 423-533 $\mathrm{nm}$ [6]. In normal use, toothbrushes alone produce essentially no wear of enamel. Even toothbrushing with toothpaste in the absence of acid causes little or no wear of enamel because, with the exception of the rarely used nonhydrated alumina, contained abrasives are softer than enamel [7].

The amount of softened enamel depends on the erosive beverage consumed by patients. Hemingway et al. [8] concluded that the amount of softened enamel removed by toothbrushing is a function of the chemical composition of the erosive medium.

Various assessment techniques have been developed to measure erosive mineral loss, such as profilometry, microradiography, scanning electron microscopy, atom force microscopy, and nanohardness and microhardness tests. Each method has its advantages and disadvantages. The main advantages of microhardness determination are the relatively low costs, the long experience with the system, and the fact that it could be combined with measurements of abrasive surface loss [9]. However, the method allows quantification of erosive tooth wear only in vitro or in situ. There are some approaches to assess erosion and abrasion in vivo, such as optical coherence tomography, but the experience with the system is very small [10].

The primary objective of the study was to assess the thickness of softened enamel removed by toothbrushing. The second objective was to compare the thickness of softened enamel produced by orange juice and citric acid. The third aim was to analyze the measurement error of the method applied for surface loss detection.

\section{Materials and methods}

\section{Preparation of enamel specimens}

Fifty caries-free human molars with no cracks on the buccal surface as viewed under the stereomicroscope (Leica, Zoom 2000 , USA; magnification $\times 25$ ) were selected from a pool of extracted teeth. The teeth were rinsed and brushed thoroughly under running tap water. Subsequently, the crowns were separated from the roots by using a diamond abrasion wheel (Isomet, 11-1180 Low Speed Saw, Buehler, USA). The teeth were then flattened on the lingual side to approximately $3 \mathrm{~mm}$ thickness using the high abrasive disk of a rotating polishing machine (Knuth-Rotor, Struers, Copenhagen, Denmark). Subsequently, each slab was embedded in resin (Paladur, Bad Homburg, Germany) in two planar parallel molds [11]. The thinner mold $(200 \mu \mathrm{m}$ thick) was then removed, and the thicker mold $(7 \mathrm{~mm}$ thick) was polished on the Knuth-Rotor polishing machine with silicone carbide paper of grain size 18,8 , and $5 \mu \mathrm{m}$ under constant tap water cooling. The embedded enamel blocks were taken out of the molds before being polished with $3 \mu \mathrm{m}$ diamond abrasive under constant cooling for 90 s (LaboPol-6, DP-Mol Polishing, DP-Stick HQ, Struers, Copenhagen, Denmark). In this manner, specimens were produced with a flat ground enamel area having suffered from a surface substance loss of $200 \mu \mathrm{m}$ at the most in the center of the window. After each polishing step, the slabs were rinsed under running tap water and sonicated for $3 \mathrm{~min}$ in deionized water. All specimens were stored in a mineral solution $\left(1.5 \mathrm{mmol} / \mathrm{L} \mathrm{CaCl}, 1.0 \mathrm{mmol} / \mathrm{L} \mathrm{KH}_{2} \mathrm{PO}_{4}\right.$, $50 \mathrm{mmol} / \mathrm{L} \mathrm{NaCl}, \mathrm{pH} 7.0$ ) when not used for experiments [12]. Prior to the experimental procedures, the specimens were further polished with $1 \mu \mathrm{m}$ diamond abrasive under constant cooling for $60 \mathrm{~s}$ (Labopol-6, DP-Mol Polishing, DP-Stick HQ, Struers, Copenhagen, Denmark).

\section{Surface microhardness measurement}

Surface microhardness measurements (SMH) were performed using a Knoop diamond under a load of 0.4903 N (MNT-10, Anton Paar, Paar Physica, Graz, Austria). Indentations were made with their long axis parallel to the vertical borders of the ground enamel area at intervals of $25 \mu \mathrm{m}$. Thus, the indentations were positioned parallel to the coronoapical axis of the teeth. The length of the indentations was measured with an optical analysis system and transferred to a computer (Leica DMR Microscope, Leica Mikroskopie and Systeme, Wetzlar, Germany). Surface microhardness was determined for allocation of the samples to the groups before the experiments and after erosion. After abrasion, the length of the indentation made after erosion was remeasured. The apparatus was recalibrated before each use.

\section{Chemical analysis}

The orange juice was analyzed for phosphorus by the ammonium molybdate method according to Chen et al. [13]. Calcium concentrations were determined by standard atomic absorption spectroscopy. The $\mathrm{pH}$ was measured with a micro glass $\mathrm{pH}$ electrode $3 \mathrm{~mm}$ in diameter (DG 101-SC, Mettler Toledo, Schwerzenbach, Switzerland). Titratable acidity and buffer capacity of orange juice and citric acid were determined by the titration of $10 \mathrm{~g}$ of test solution with $0.5 \mathrm{~N} \mathrm{NaOH}$. Buffer capacity $\beta$, in millimoles per liter $\mathrm{pH}$ was calculated as $\beta=-\Delta C / \Delta \mathrm{pH}$ where $\Delta C$ is the amount of titrator used (base) and $\Delta \mathrm{pH}$ is the change in $\mathrm{pH}$ caused by the addition of the titrator. All measurements were made in duplicate [14].

Surface softening

Each specimen was immersed in $50 \mathrm{~mL}$ of orange juice (Granini, Eckes-Granini SA, Henniez, Switzerland) or in 
Table 1 Phosphate and calcium concentrations, amount of base necessary to raise the $\mathrm{pH}$ to $3.6,5.5$, and 7.0, and buffer capacity at the respective $\mathrm{pH}$ in orange juice and citric acid

\begin{tabular}{lcccccccc}
\hline $\begin{array}{l}\text { Erosive } \\
\text { medium }\end{array}$ & $\begin{array}{l}\text { Phosphate } \\
{[\mathrm{mmol} / \mathrm{L}]}\end{array}$ & $\begin{array}{l}\text { Calcium } \\
{[\mathrm{mmol} / \mathrm{L}]}\end{array}$ & $\begin{array}{l}\mathrm{ml} \mathrm{NaOH} \\
{[0.5 \mathrm{~N}] \text { to }} \\
\mathrm{pH} 3.6\end{array}$ & $\begin{array}{l}\text { Buffer capacity pH3.6 } \\
[\mathrm{mmol} / \mathrm{L} \mathrm{pH})]\end{array}$ & $\begin{array}{l}\mathrm{ml} \mathrm{NaOH} \\
{[0.5 \mathrm{~N}] \text { to }} \\
\mathrm{pH} 5.5\end{array}$ & $\begin{array}{l}\text { Buffer capacity pH5.5 } \\
{[\mathrm{mmol} /(\mathrm{L} \mathrm{pH})]}\end{array}$ & $\begin{array}{l}\mathrm{ml} \mathrm{NaOH} \\
{[0.5 \mathrm{~N}]} \\
\mathrm{pH} 7.0\end{array}$ & $\begin{array}{l}\mathrm{Buffer} \text { capacity pH7.0 } \\
{[\mathrm{mmol} /(\mathrm{L} \mathrm{pH})]}\end{array}$ \\
\hline Citric acid & 0 & 0 & 0.04 & 23.69 & 0.98 & 22 & 1.36 & 3.42 \\
Orange juice & 1.58 & 2.29 & 0.07 & 48.64 & 1.58 & 28 & 2.27 & 5.43 \\
\hline
\end{tabular}

citric acid for 3 min under constant agitation $(75 \mathrm{rpm})$ on an orbital shaker (Salvis AG, Reussbühl, Switzerland) at $37^{\circ} \mathrm{C}$. The $\mathrm{pH}$ of citric acid (adjusted with $\mathrm{NaOH}$ ) was 3.6 and its concentration was $0.0341 \mathrm{mmol} / \mathrm{L}(0.65 \%)$, which was equivalent to the citric acid in the orange juice under study. The specimens were then carefully rinsed with deionized water and dried for $5 \mathrm{~s}$ with oil-free air.

\section{Toothbrush abrasion}

The specimens were brushed in an automatic brushing machine (Syndicad Ingenieurbüro, München, Germany) either with slurry or artificial saliva $(0.381 \mathrm{~g} \mathrm{NaCl}, 0.213 \mathrm{~g}$ $\mathrm{CaCl}_{2} \cdot 2 \mathrm{H}_{2} \mathrm{O}, 1,114 \mathrm{~g} \mathrm{KCl}, 0.738 \mathrm{~g} \mathrm{KH}_{2} \mathrm{PO}_{4}$, and 2,200 $\mathrm{g}$ mucin in 1,000 $\mathrm{mL}$ distilled water; $\mathrm{pH} 7$ ) [15]. Surface loss was measured after every 30 single brushing strokes up to 390 and then after every 100 strokes up to 590. The manual toothbrush (Ultra super sensitive, Trisa, Triengen, Switzerland) loaded with $150 \mathrm{~g}$ was used. Toothpaste slurry was prepared with a fluoridated dentifrice (Sensodyne Proschmelz, GlaxoSmithKline, Consumer Healthcare AG, Münchenbuchsee, Switzerland $\left[\mathrm{RDA}=30-40 ; 1450 \mathrm{~F}^{-}\right.$; $\mathrm{pH} 6.54$; silica abrasive]) and artificial saliva in a weight ratio of 1:3. After brushing, the specimens were carefully rinsed with deionized water and dried for $5 \mathrm{~s}$ with oil-free air.

\section{Measurement of toothbrush abrasion}

The detection of enamel abrasion was based on SMH by calculating the depth of the indentations. The difference between the depth after erosion and the depth after abrasion of the same indentation provided a direct measure for the loss of substance by abrasion at this site [5]. The depths of the indentations were calculated from their lengths using the geometrical formula: $d=(L / 2) \times \tan 3.75^{\circ}$ (where $d$ is the depth and $L$ is the length of indentation).

\section{Study design}

Three (pre-experimental) indentations were made in the center of the enamel window of each prepared specimen and the average SMH was calculated, making the tooth the statistical unit. Based on these data, the specimens were assigned to ten vials according to the distribution of all specimens. Then, one specimen from each of the ten vials was randomly selected and assigned to an experimental group in a randomized block design [5]. The treatment groups were as follows:

1. citric acid, abrasion with toothpaste slurry $(n=10)$;

2. citric acid, abrasion with artificial saliva $(n=10)$;

3. orange juice, abrasion with toothpaste slurry $(n=10)$;

4. orange juice, abrasion with artificial saliva $(n=10)$;

5. no softening, abrasion with toothpaste slurry $(n=10)$.

SMH (six indentations) were conducted on enamel specimens before and after erosive challenge. The array was such that the corresponding indentations were neighboring within a distance of $25 \mu \mathrm{m}$. After toothbrush abrasion, the indentations after softening were located and surface loss was calculated.

\section{Statistics}

Since the difference between groups 2, 4, and 5 was negligible, statistical comparison of groups 1 and 3 was of great interest. To analyze the effect of the group (citric acid, abrasion with slurry vs. orange juice, abrasion with slurry), the effect of the number of brushing strokes, and the interaction of the group and the number of strokes, a nonparametric model for longitudinal data after Brunner, Domhof, and Langer [16] was applied. An interaction effect is present if the effect of the number of strokes is different in both groups. All computations were made using SAS 9.1.3 (SAS Institute, Cary, NC, USA). The significance level was set at 0.05 .

\section{Statistical evaluation of measurement error}

In order to report the measurement error, the British Standards Institution [17] recommended the repeatability coefficientthe maximum difference likely to occur between two successive measurements. The best way to examine repeatability is to take repeated measurements on a series of indentations [18]. Since the same method is used for the repeated measurements, the mean difference should be zero. The Bland and Altman plot was used to assess the repeatability of the method by comparing repeated measure- 
ments. The repeatability coefficient was read from the Bland and Altman plot (Medical for Windows, version 9.3.0.0, Mariakerke, Belgium).

\section{Results}

The results of the chemical analysis are presented in Table 1. Total enamel losses by toothbrush abrasion as well as the lower and upper bounds of the 95\% confidence interval $(95 \% \mathrm{CI})$ in groups are presented in Table 2.

For groups 1 and 3, an increase of enamel loss with increasing brushing strokes was observed. No increase was observed after the application of 240 single brushing strokes with toothpaste slurry, indicating that the softened layer was removed nearly completely. The calculated thickness of the softened enamel amounted to 254$323 \mathrm{~nm}$. The effect of the group (citric acid, abrasion with slurry vs. orange juice, abrasion with slurry), the effect of the number of brushing strokes, and the interaction of the group and the number of strokes were significant to the nominal level 0.05 with $p$ value $<0.00001$.

Negligible abrasion [mean $(95 \% \mathrm{CI})]$ was found after 590 single brushing strokes in groups $2[16 \mathrm{~nm}(5-$ $27 \mathrm{~nm})], 4[14 \mathrm{~nm}(5-23 \mathrm{~nm})]$, and 5 [28 nm $(10-$ $46 \mathrm{~nm})]$.

Analyses of measurement error revealed that the two measurements on the depth of the same indentation did not differ by more than $12 \mathrm{~nm}$ before softening, $20 \mathrm{~nm}$ after softening, and $55 \mathrm{~nm}$ after abrasion, respectively.

\section{Discussion}

The present results indicate that the amount of softened enamel removed by toothbrushing depends on the erosive medium used. This corresponds well with the study of Hemingway [8] where abrasive enamel loss differed between the drinks and was positively correlated with $\mathrm{pH}$ and calcium concentration, but not phosphate concentration or titratable acidity. There is no direct evidence why softening produced by citric acid is greater than that of orange juice as the concentration of citric acid was equivalent in both solutions. It could be speculated that citrate ions bind calcium in orange juice first and afterwards attack the calcium of enamel. However, one should keep in mind that the chelating effect in the $\mathrm{pH}$ used was negligible. The orange juice had no additional calcium and was undersaturated with respect to hydroxyapatite and enamel. The greater softening in citric acid compared to orange juice can, therefore, not be explained

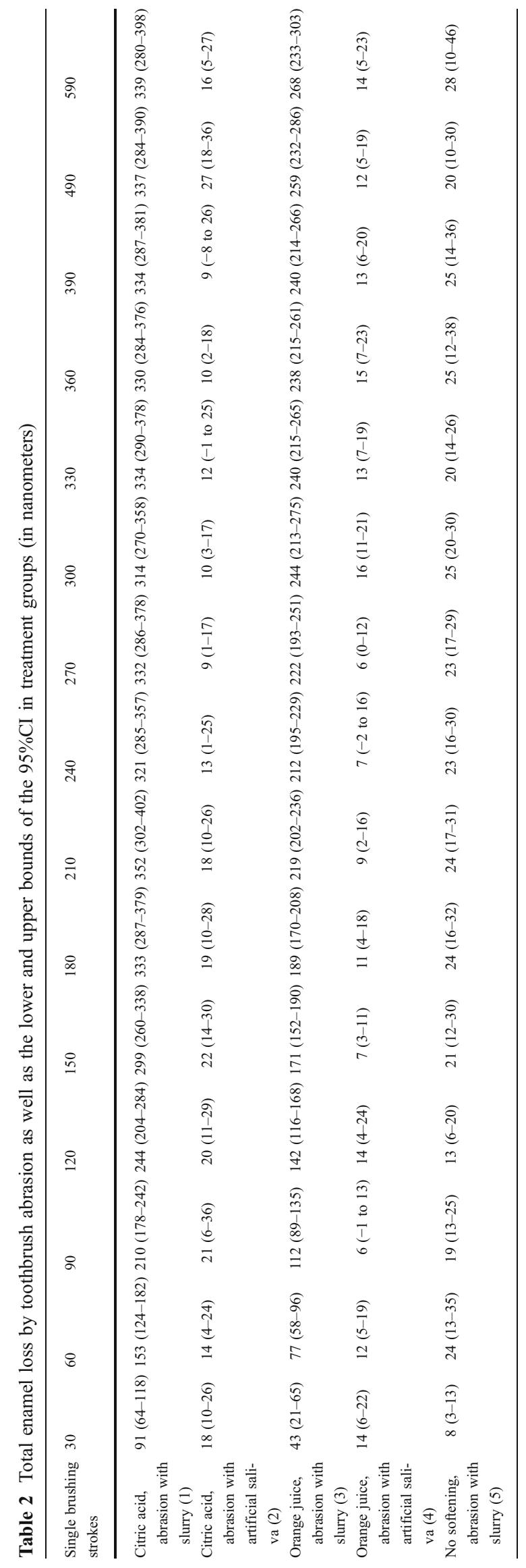


by the calcium content but rather with the interaction of orange juice components and toothpaste slurry on the enamel surface. Obviously, the effect of citric acid differs from that of orange juice. Thus, the softening of the enamel surface with citric acid should be cautiously extrapolated to the other soft drinks. There is a need of an erosive equivalent for experiments on erosion in vitro in order to be able to compare the studies of different research groups. In this study, citric acid was chosen because of its widespread use as an additive to foodstuffs or drinks (e.g., orange juice).

In erosion, the amount of mineral dissolved from enamel depends on a number of conditions: $\mathrm{pH}$, the buffering effect or the concentration of acids, and the length of the exposure time. Finally, the presence of suitable concentrations of calcium, phosphate, and fluoride in the drink may counteract the dissolution [19]. Dental mineral is dissolved layer by layer, leaving the subsurface enamel almost unaltered. Researchers have arrived at different results regarding the thickness of the layer of softened enamel, varying between 0.2 and $5 \mu \mathrm{m}[5,6,20,21]$. It could be hypothesized that it is due to a different erosive medium being used or in the specimens' preparation. It is unclear whether some softened enamel remains after abusive toothbrushing. Unfortunately, the present technique does not allow assessing whether the entire softened layer was removed. It is speculated that the softened layer removed with toothbrushing represents the major part of the total mineral loss. However, as visual diagnosis of erosion is quite difficult (may hardly detect involvement of dentine) [22], monitoring the thickness of the softened layer using optical coherence tomography could be a good opportunity for diagnostic purposes.

No increase was observed after the application of 240 single brushing strokes with toothpaste slurry, indicating that the demineralized layer was removed nearly completely. Interestingly, there was a negligible abrasion in the group where softened enamel was brushed with artificial saliva. From this finding, it could be concluded that softened enamel will not be removed by brushing without toothpaste. This corresponds well with the study of Lippert et al. [23] where the typical prismatic structure of demineralized enamel was still intact after $30 \mathrm{~s}$ of power toothbrushing of specimens that were previously stored in citric acid $(\mathrm{pH}$ 3.25 ) for 1-3 min. It is also consistent with the study of Voronets et al. [24] where the loss of softened enamel produced with a soft toothbrush with artificial saliva amounted to $28 \mathrm{~nm}$, which is not much different from the findings of the present study. In group 5, the loss of softened enamel only happened during the first 60 brushing strokes. From there on, no changes were documented. Thus, it seems as if the first strokes removed more material than the following ones. This increase of abrasion at the beginning may not be overinterpreted. The inhomogeneity of the data is limited within the measurement error, which is
$55 \mathrm{~nm}$ after abrasion. As there was no abrasion found when the enamel was not softened and the specimens were brushed only with artificial saliva in the preliminary experiments and the literature [7], this group was not included in this model.

In order to report the measurement error, the repeatability coefficient was used. Several measurements of the same quantity on the same subject will not, in general, be the same. Repeated measurements on the same subject will vary around the true value because of measurement error. Measurement error is a result of a measurement minus a true value. In general, the error of measurement is unknown because a true value is unknown. However, the uncertainty of the result of a measurement may be evaluated. There are few ways of reporting the error. However, the British Standards Institution [17] recommended the repeatability coefficient - the maximum difference likely to occur between two successive measurements. The difference is numerically unimportant, but tells us how much the two measurements on the same subject are unlikely to be apart. However, by using properly maintained and calibrated equipment, trained personnel, and appropriate testing environments, testing error and variability can be minimized. One should keep in mind that, due to the inhomogeneity of the data with broad confidence intervals, the method is debatable with respect to determination of minimal amounts of wear as needed for testing the abrasivity of different toothpastes with similar abrasivity values.

One should keep in mind that a number of abrasion conditions (brushing force and speed should be controlled, the homogeneity of toothpaste slurry should be kept) should be fulfilled [25]. In this model, e.g., the slurry homogeneity was maintained in a way that the slurry was often changed and the vials were moved. It is important to prevent sinking of toothpaste particles. For this purpose, Joiner et al. [26] suggested the use of sodium carboxymethyl cellulose for slurry preparation.

Obviously, more research is needed to evaluate the relationship between erosive beverages and abrasivity of different toothpastes.

Acknowledgement The authors wish to thank Stefanie Hayoz and Prof. J. Huesler for the statistical advice.

Conflicts of interest The authors declare that they have no conflicts of interest.

\section{References}

1. Ten Cate JM, Imfeld T (1996) Dental erosion, summary. Eur J Oral Sci 104:241-244

2. Featherstone JDB, Lussi A (2006) Understanding the chemistry of dental erosion. In: Lussi A (ed) Dental erosion. Karger, Basel, pp 66-76 
3. Ten Cate JM, Larsen MJ, Pearce EIF, Fejerskov O (2003) Chemical interactions between the tooth and oral fluids. In: Fejerskov O, Kidd E (eds) Dental caries: the disease and its clinical management. Blackwell Munksgaard, Oxford, pp 59-65

4. Dawes C (2003) What is the critical pH and why does a tooth dissolve in acid? J Can Dent Assoc 69:722-724

5. Jaeggi T, Lussi A (1999) Toothbrush abrasion of erosively altered enamel after intraoral exposure to saliva: an in situ study. Caries Res 33:455-461

6. Wiegand A, Wegehaupt F, Werner C, Attin T (2007) Susceptibility of acid-softened enamel to mechanical wear-ultrasonication versus toothbrushing abrasion. Caries Res 41:56-60

7. Addy M (2005) Tooth brushing, tooth wear and dentine hypersensitivity - are they associated? Int Dent J 55:261-267

8. Hemingway CA, Parker DM, Addy M, Barbour ME (2006) Erosion of enamel by non-carbonated soft drinks with and without toothbrushing abrasion. Br Dent J 201:447-450

9. Attin T (2006) Methods for assessment of dental erosion. In: Lussi A (ed) Dental erosion. Karger, Basel, pp 152-172

10. Otis LL, Everett MJ, Sathyam US, Colston BW (2000) Optical coherence tomography: a new imaging technology for dentistry. J Am Dent Assoc 131:511-514

11. Lussi A, Jaeggi T, Schaerer S (1993) The influence of different factors on in vitro enamel erosion. Caries Res 27:387-393

12. Zero DT, Rahbek I, Fu J, Proskin HM, Featherstone JDB (1990) Comparison of the iodide permeability test, the surface microhardeness test and mineral dissolution of bovine enamel following acid challenge. Caries Res 24:181-188

13. Chen PS, Toribara TY, Warner H (1956) Microdetermination of phosphorus. Anal Chem 28:1756-1758

14. Lussi A, Hellwig E (2001) Erosive potential of oral care products. Caries Res 35:52-56

15. Newby CS, Creeth JE, Rees GD, Schemehorn BR (2006) Surface microhardness changes, enamel fluoride uptake, and fluoride availability from commercial toothpastes. J Clin Dent 17:94-99

16. Brunner E, Domhof S, Langer F (2002) Nonparametric analysis of longitudinal data in factorial designs. Wiley, New York

17. British Standards Institution (1979) Precision of test methods. Guide for the determination and reproducibility for a standard test method (BS 5497, part 1). British Standards Institution, London

18. Bland JM, Altman DG (1986) Statistical methods for assessing agreement between two methods of clinical measurement. Lancet $1: 307-310$

19. Larsen MJ, Nyvad B (1999) Enamel erosion by some soft drinks and orange juices relative to their $\mathrm{pH}$, buffering effect and contents of calcium phosphate. Caries Res 33:81-87

20. Attin T, Buchalla W, Gollner M, Hellwig E (2000) Use of variable remineralization periods to improve the abrasion resistance of previously eroded enamel. Caries Res 34:48-52

21. Eisenburger M, Addy M (2003) Influence of liquid temperature and flow rate on enamel erosion and surface softening. J Oral Rehab 30:1076-1080

22. Ganss C, Klimek J, Lussi A (2006) Accuracy and consistency of the visual diagnosis of exposed dentine on worn occlusal/incisal surfaces. Caries Res 40:208-212

23. Lippert F, Parker DM, Jandt KD (2004) Toothbrush abrasion of surface softened enamel studied with tapping mode AFM and AFM nanoindentation. Caries Res 38:464-472

24. Voronets J, Jaeggi T, Buergin W, Lussi A (2008) Controlled toothbrush abrasion of softened human enamel. Caries Res 42:286-290

25. Parry J, Harrington E, Rees GD, McNab R, Smith AJ (2008) Control of brushing variables for the in vitro assessment of toothpaste abrasivity using a novel laboratory model. J Dent 36:117-124

26. Joiner A, Schwarz A, Philpotts CJ, Cox TF, Huber K, Hannig M (2008) The protective nature of pellicle towards toothpaste abrasion on enamel and dentine. J Dent 36:360-368 